NIKHEF/2011-020

July 2011

\title{
Gravity on a Little Warped Space
}

\author{
Damien P. George ${ }^{(a)}$.1 and Kristian L. McDonald ${ }^{(b)}$ 2 \\ (a) Nikhef Theory Group, Science Park 105, \\ 1098 XG Amsterdam, The Netherlands \\ (b) Max-Planck-Institut für Kernphysik, \\ Postfach 1039 80, 69029 Heidelberg, Germany.
}

\begin{abstract}
We investigate the consistent inclusion of 4D Einstein gravity on a truncated slice of $A d S_{5}$ whose bulk-gravity and UV scales are much less than the 4 D Planck scale, $M_{*} \ll$ $M_{P l}$. Such "Little Warped Spaces" have found phenomenological utility and can be motivated by string realizations of the Randall-Sundrum framework. Using the interval approach to brane-world gravity, we show that the inclusion of a large UV-localized Einstein-Hilbert term allows one to consistently incorporate 4D Einstein gravity into the low-energy theory. We detail the spectrum of Kaluza-Klein metric fluctuations and, in particular, examine the coupling of the little radion to matter. Furthermore, we show that Goldberger-Wise stabilization can be successfully implemented on such spaces. Our results demonstrate that realistic low-energy effective theories can be constructed on these spaces, and have relevance for existing models in the literature.
\end{abstract}

\footnotetext{
${ }^{1}$ Email: dpgeorge@nikhef.nl

${ }^{2}$ Email: kristian.mcdonald@mpi-hd.mpg.de
} 


\section{Introduction}

The Randall-Sundrum (RS) framework provides a natural means by which to generate hierarchically separated, radiatively-stable mass scales [1]. As such, it has received much attention in connection with the gauge hierarchy problem of the Standard Model (SM). In its standard incarnation, the ultraviolet (UV) scale of a warped extra dimension is (approximately) identified with the 4D Planck scale, while the infrared (IR) scale is associated with electroweak symmetry breaking (i.e. the weak scale). The hierarchy between these two scales is generated by spacetime warping, resulting in a mechanism for realizing the weak scale from Planck-scale sized input parameters.

Of course, any solution to the gauge hierarchy problem should explain why the weak scale is radiatively stable relative to some UV cutoff, $M_{*} \gtrsim \mathrm{TeV}$. However, this cutoff need not be the Planck scale and may instead be some intermediate scale at which new physics arises in connection with, e.g., baryogenesis, flavor, etc. This is precisely the philosophy of the Little Randall-Sundrum (LRS) model [2, 3, 4], which employs a Little Warped Space (LWS) [5, 6] - namely a truncated slice of $A d S_{5}$ whose bulk-gravity and UV scales are much less than the 4D Planck mass, $M_{*} \ll M_{P l}$ - to realize a candidate solution to the hierarchy problem. This framework provides an explanation for the stability of the weak scale but only relative to some intermediate scale $M_{*}$, beyond which a UV completion is expected.

If the UV scale of a warped space is decoupled from the 4D Planck scale, an evident question arises: How does one include 4D Einstein gravity in such a low energy effective theory framework? A necessary ingredient for any realistic low energy theory is that it should reproduce 4D Einstein gravity at large distances, a point that must also hold for models constructed on a LWS. Despite the fact that such theories are not intended to explain the full weak/Planck hierarchy, it should be possible to incorporate 4D gravity if the effective theory framework is to be valid. To date, this matter has not been addressed in the literature 1 It is the goal of the present work to study the consistent inclusion of $4 \mathrm{D}$ gravity in models constructed on a LWS, and to detail the spectrum of physical metric fluctuations obtained once $4 \mathrm{D}$ gravity is included.

As suggested in Ref. [5], the key ingredient that enables 4D Einstein gravity to be realized on a LWS is the inclusion of a large UV-localized Einstein-Hilbert term 2 Large branelocalized curvature terms were originally studied by Dvali, Gabadadze, and Porrati (DGP) [8] in the context of a flat extra dimension, and have a rich history in the literature. A number of works have studied the issues of stability and consistency that arise in the presence of a DGP term, and some subtleties have been found [9, 10, 11, 12] concerning, e.g., the onset of strong coupling among metric fluctuations. However, it is known that no such strong coupling problems occur when a large UV localized DGP term is introduced in warped models, at least for energies below the original UV cutoff of the theory [13].

There exists an additional formal reason to consider the effects of a large UV localized

\footnotetext{
${ }^{1}$ A previous work has embedded the LRS model in an extended spacetime [7].

${ }^{2}$ Equivalently, a UV-localized "brane curvature" or "DGP" term. We use these labels for the UV term interchangeably throughout this work.
} 
Einstein-Hilbert term in warped models. As is well known, strongly warped regions that admit an RS-like description can arise naturally in string theory flux compactifications [14]. Interestingly, UV-localized curvature terms that are parametrically large relative to the effective UV scale can easily arise in the string constructs [15]. This suggests that the approach of the LRS model [2] and the Mini-Seesaw [5, 6] (which also employs a LWS) may be well motivated from a "top-down" perspective. However, the broader study of phenomenological models on a LWS requires one to understand the gravitational sector of these spaces in detail, and to demonstrate their stability. Our aim is to carry out these analyses and thus demonstrate conclusively that viable low energy models which include Einstein gravity can be realized.

Throughout this work we consider warped spaces with a low UV scale, $M_{*} \ll M_{P l}$, but for the most part we will not specify the particular value of $M_{*}$. Previous works employing a LWS have taken the UV scale to be significantly lighter than the Planck scale; in the case of the LRS model one has $M_{*} \sim 10^{3} \mathrm{TeV}$, while in Refs. [5, 6] the UV scale is taken at the $\mathrm{TeV}$ scale (with the warping employed to generate a light hidden sector scale). Our analysis applies to these works, but applies more generally to models where the hierarchy between $M_{*}$ and $M_{P l}$ is not so severe. For example, our results would be relevant for models with a UV scale of $M_{*} \sim 10^{14} \mathrm{GeV}$ in connection with, e.g., a Grand Unification scale. Such models still require a parametrically large UV curvature term to reproduce viable 4D gravity, so the relation $M_{*} \ll M_{P l}$ still holds. Given that string realizations of RS models readily generate large UV curvature terms [15], it would seem prudent to consider the UV scale of the RS framework as a free parameter that may or may not be directly connected to the 4D Planck scale, and to study the phenomenological consequences of its variation. Our results remain relevant in this context.

The layout of this work is as follows. In Sec. 2 we describe the setup for our analysis and derive the spectrum of spin-two Kaluza-Klein (KK) modes in the presence of the large UV Einstein-Hilbert term. We focus on the radion in Secs. 3 and 4, where we, respectively, obtain the precise form of the physical gravi-scalar fluctuation in the presence of the UV term, and consider the coupling of the "little radion" to matter. In Sec. 5 we show that the length of a LWS (with large UV brane term) is readily stabilized by implementing the Goldberger-Wise mechanism [16]. We obtain the radion mass and discuss the coupling of the radion to matter for the stabilized setup. A brief discussion on the relevance of our results for the LRS model and the Mini-Seesaw3 appears in Sec. 6, and Sec. 7 attempts to shed light on some of our findings via the AdS/CFT correspondence. We conclude in Sec. 8 and some technical details are given in an Appendix.

\footnotetext{
${ }^{3}$ Our results have particular relevance for the latter work, as we demonstrate the suppressed coupling of KK gravitons and the radion to the UV localized SM, and thus prove phenomenological viability of the gravitational sector of this model.
} 


\section{Gravity in a Little Warped Space}

We consider a truncated warped space whose bulk-gravity and UV scales are much less than the 4D Planck mass, $M_{*} \ll M_{P l}$, namely a Little Warped Space [5, 6]. To study the effects of a large UV curvature term on the spectrum of metric fluctuations we will employ the interval approach to brane-world gravity [17, 18, 19]. This approach, which can reproduce standard RS results obtained on an orbifold, enables a transparent treatment of boundary curvature terms; these simply modify the form of the boundary Einstein equations, and thus modify the boundary conditions (BCs) for the metric fluctuations. Before proceeding, we note that previous works have considered the effects of brane curvature terms in the RS framework using the orbifold picture [20, 21], and for $A d S_{5} / A d S_{4}$ in the interval approach [18, 19].

We consider a warped extra dimension, described by the coordinate $y \in[0, L]$, such that a UV (IR) brane of characteristic energy scale $M_{*}\left(e^{-k L} M_{*}\right)$ is located at $y=0(y=L)$. The metric is given by

$$
d s^{2}=e^{-2 k y} \eta_{\mu \nu} d x^{\mu} d x^{\nu}+d y^{2}=G_{M N} d x^{M} d x^{N},
$$

where $M, N, . .(\mu, \nu, .$.$) are the 5 \mathrm{D}(4 \mathrm{D})$ Lorentz indices and $k$ is the $A d S_{5}$ curvature. The action for the LWS, with brane localized curvature terms included, is

$$
\begin{aligned}
S=\int_{\mathcal{M}} d^{5} x \sqrt{-G}\left\{2 M_{*}^{3} \mathcal{R}-\Lambda\right\}+\sum_{i} \int d^{4} x \sqrt{-g}\left\{M_{i}^{2} R-V_{i} / 2\right\} \\
+4 M_{*}^{3} \oint_{\partial \mathcal{M}} \sqrt{-g} K
\end{aligned}
$$

where $\mathcal{R}$ is the bulk Ricci scalar constructed from $G_{M N}$ and $M_{*}$ is the $5 \mathrm{D}$ gravity scale. The brane localized curvature $R$ has coefficient $M_{i}$ on the $i$ th boundary $(i=0, L)$ and is constructed with $g_{\mu \nu}$ (the restriction of $G_{\mu \nu}$ to the relevant boundary). The last term is the usual Gibbons-Hawking boundary term [22], with $K$ being the extrinsic curvature of the manifold $\mathcal{M}$. This term is necessary to obtain consistent Einstein equations on an interval [17]. The gravitational field is sourced by the bulk cosmological constant $\Lambda$ and brane tensions $V_{i}$. The bulk curvature is $k=\sqrt{-\Lambda / 24 M_{*}^{3}}$ and the brane tensions take their usual RS values, $V_{i}=-24 k M_{*}^{3} \theta_{i}$, with $\theta_{0}=-\theta_{L}=-1$. We will employ the rescaled dimensionless variables $v_{i}=M_{i}^{2} k / M_{*}^{3}$ and $w_{i}=V_{i} / 2 M_{*}^{3} k$ for the brane quantities.

The effective 4D Planck mass has contributions from both the bulk and brane intrinsic curvatures, and is calculated as

$$
M_{P l}^{2}=\frac{M_{*}^{3}}{2 k}\left\{1-e^{-2 k L}+v_{0}+v_{L} e^{-2 k L}\right\} .
$$

We will be interested in the limit $M_{0} \gg M_{*}$, corresponding to $v_{0} \gg 1$. In this limit the 4D Planck mass is predominantly determined by the coefficient of the UV Einstein-Hilbert term:

$$
M_{P l}^{2} \simeq \frac{M_{*}^{3}}{2 k} \times v_{0}=\frac{M_{0}^{2}}{2}
$$


Thus, provided $M_{0}$ is suitably large, a viable $4 \mathrm{D}$ Planck mass can be obtained even when the bulk gravity scale is much less than the Planck scale, $M_{*} \ll M_{P l}$. This is the main reason to introduce the large UV term. In addition to modifying the 4D Planck mass, this term, in general, modifies the properties of the metric fluctuations, including the KK gravitons and the radion. Our goal is to detail the spectrum of these modes and consider the effects of the large UV term on the low energy particle (KK) spectrum.

Varying the action gives the bulk equations of motion,

$$
\mathcal{R}_{M N}-\frac{1}{2} G_{M N} \mathcal{R}=-\frac{\Lambda}{4 M_{*}^{3}} G_{M N} .
$$

The boundary conditions are obtained by combining the variations of the 4D brane action and the Gibbons-Hawking term with the surface terms arising from the variation of the bulk action. The result is [18]

$$
\left[\frac{v_{i}}{k}\left(R_{\mu \nu}-\frac{1}{2} g_{\mu \nu} R\right)+\frac{1}{2} g_{\mu \nu} k w_{i}+\theta_{i} \sqrt{G^{55}}\left(g_{\mu \nu, 5}-g_{\mu \nu} g_{\alpha \beta, 5} g^{\alpha \beta}\right)\right]_{y=y_{i}}=0 .
$$

The notation here is that the above equation must be evaluated separately at $y=0, L$. Without loss of generality we work in a "straight" gauge defined by $G_{\mu 5}=0$ [18]. Expanding the metric in terms of a fluctuation, $G_{M N}=G_{M N}^{0}+h_{M N}$, where the zeroth order metric is given by $G_{\mu \nu}^{0}=e^{-2 k y} \eta_{\mu \nu}, G_{55}^{0}=1$ and $G_{M 5}^{0}=h_{M 5}=0$ in a straight gauge, the boundary condition can be expressed as

$$
\begin{array}{r}
{\left[\frac{v_{i}}{2 k}\left\{h_{\alpha \mu, \nu}{ }^{\alpha}+h_{\alpha \nu, \mu}^{\alpha}-h_{\mu \nu, \alpha}^{\alpha}-\tilde{h}_{, \mu \nu}-g_{\mu \nu}\left(h_{\alpha \beta,}{ }^{\alpha \beta}-\tilde{h}_{, \alpha}^{\alpha}\right)\right\}\right.} \\
\left.+\theta_{i}\left\{2 k h_{\mu \nu}+h_{\mu \nu, 5}-g_{\mu \nu} \tilde{h}_{, 5}-3 k g_{\mu \nu} h_{55}\right\}\right]_{y=y_{i}}=0
\end{array}
$$

where indices are raised with $g^{\mu \nu}=e^{2 k y} \eta^{\mu \nu}$.

In a straight gauge we can always use remnant gauge freedom to write the metric fluctuation $h_{55}$ as [18, 19]

$$
h_{55}\left(x^{\mu}, y\right)=F(y) \psi\left(x^{\mu}\right)
$$

where $F(y)$ is an arbitrary function of $y$ satisfying $\int_{0}^{L} d y F(y) \neq 0$. One can always recast an arbitrary $h_{55}$ into the form (8) by performing a general 5D coordinate transformation $x^{M} \rightarrow x^{M}+\xi^{M}$ with $\xi^{\mu}=0$ and [18, 19]

$$
\xi^{5}=\frac{1}{2} \int^{y} d y h_{55}-\frac{1}{2} \int^{y} d y F(y) \psi
$$

The ability to specify an arbitrary $F(y)$ is a remnant gauge freedom.

For massive $4 \mathrm{D}$ modes the tensor $h_{\mu \nu}$ can be written as

$$
h_{\mu \nu} \rightarrow h_{\mu \nu}+\partial_{\mu} V_{\nu}+\partial_{\nu} V_{\mu}+e^{-2 k y} \partial_{\mu} \partial_{\nu} \mathcal{S}_{1}+G_{\mu \nu}^{0} \mathcal{S}_{2}
$$


where $h_{\mu \nu}$ is now transverse-traceless with five degrees of freedom, $\partial^{\alpha} h_{\alpha \beta}=\eta^{\alpha \beta} h_{\alpha \beta}=0$, and $V_{\mu}$ is transverse, $\partial^{\alpha} V_{\alpha}=0 . \mathcal{S}_{1}$ and $\mathcal{S}_{2}$ are scalar degrees of freedom. One can show that the physical massive modes are contained in $h_{\mu \nu}$. This has been discussed in detail in [18, 19] and we have verified their results. Here we simply note a few key points (using the notations of [18] so the reader can readily find more details there). One can use the bulk equations of motion to show that $\partial_{5}\left(e^{2 k y} \bar{V}_{\mu}\right)=0$, which fixes the $y$-dependence of $V_{\mu}$ as $e^{-2 k y}\left(\bar{V}_{\mu}\right.$ is the Fourier transform of $V_{\mu}$ ). Thus one can use the transverse part of the remaining $\xi^{\mu}$ gauge freedom to remove $V_{\mu}$. The longitudinal part of the $\xi^{\mu}$ gauge freedom can be used to remove one scalar degree of freedom so that $\mathcal{S}_{1}$ and $\mathcal{S}_{2}$ can be expressed in terms of $\psi$ and an unspecified function $f_{1}(x)$. For massive modes the boundary conditions can be shown to require 4

$$
\left(\theta_{i}-v_{i}\right) \times\left[\frac{e^{-2 k y} \bar{f}_{1}}{\eta^{\mu \nu} p_{\mu} p_{\nu}}+\mathcal{F} \bar{\psi}\right]_{y=y_{i}}=0
$$

where $\mathcal{F}^{\prime}=F$. Thus the boundary conditions force $\bar{f}_{1}=\bar{\psi}=0$, assuming the branecurvature coefficients are not tuned to satisfy $\theta_{i}=v_{i}$. Together, this shows that the only physical, massive degrees of freedom are contained in $h_{\mu \nu}$.

KK expanding the physical fluctuations as

$$
h_{\mu \nu}(x, y)=\kappa_{*} \sum_{n} h_{\mu \nu}^{(n)}(x) f_{n}(y)
$$

where $\kappa_{*}$ is chosen to give the $4 \mathrm{D}$ fields $h_{\mu \nu}^{(n)}$ a canonical mass dimension, the bulk equations of motion reduce to

$$
\left(\partial_{5}^{2}+e^{2 k y} m_{n}^{2}-4 k^{2}\right) f_{n}(y)=0
$$

giving

$$
f_{n}(y)=\frac{1}{N_{n}}\left\{J_{2}\left(\frac{m_{n}}{k} e^{k y}\right)+\beta_{n} Y_{2}\left(\frac{m_{n}}{k} e^{k y}\right)\right\}
$$

Here, $m_{n}$ is the mass of the $n^{\text {th }} \mathrm{KK}$ mode. The boundary conditions require

$$
\left.\left[\partial_{5}+2 k-e^{2 k y} m_{n}^{2} \frac{\lambda_{i}}{2 \theta_{i}}\right] f_{n}(y)\right|_{y=y_{i}}=0
$$

enforcing which gives

$$
\beta_{n}=-\frac{J_{1}\left(z_{i}\right)-z_{i} v_{i} \theta_{i} / 2 J_{2}\left(z_{i}\right)}{Y_{1}\left(z_{i}\right)-z_{i} v_{i} \theta_{i} / 2 Y_{2}\left(z_{i}\right)}
$$

with $z_{i}=m_{n} e^{k y_{i}} / k$. Correcting for the different definitions of the parameters in the action, this result agrees with that obtained via the orbifold approach in Ref. [20] (see Eq. (2.9) in the published version).

\footnotetext{
${ }^{4}$ The "bars" again denote 4D Fourier transforms.
} 
The KK spectrum is found by equating the values of $\beta_{n}$ obtained at the distinct boundaries. For comparison, note that the usual KK graviton spectrum in RS models is determined by [25]

$$
\beta_{n}^{R S}=-\frac{J_{1}\left(z_{0}\right)}{Y_{1}\left(z_{0}\right)}=-\frac{J_{1}\left(z_{L}\right)}{Y_{1}\left(z_{L}\right)},
$$

corresponding to Neumann BCs at both the IR and UV branes. The usual RS KK masses are therefore (approximately) determined by the solutions to $J_{1}\left(m_{n}^{R S} e^{k L} / k\right)=0$. In the limit of a large UV-brane curvature term, $v_{0} \gg 1$, with $v_{L}=0$ for simplicity, Eq. (16) gives

$$
\beta_{n}=-\frac{J_{1}\left(z_{L}\right)}{Y_{1}\left(z_{L}\right)} \simeq-\frac{J_{2}\left(z_{0}\right)}{Y_{2}\left(z_{0}\right)} .
$$

The UV BC is changed from Neumann to (approximately) Dirichlet for $v_{0} \gg 1$, while the IR $\mathrm{BC}$ is unchanged 5 For KK modes with mass $m_{n} / k \ll 1$ the spectrum remains determined by the solutions to $J_{1}\left(m_{n} e^{k L} / k\right)=0$. Light $\mathrm{KK}$ modes are localized towards the IR brane and are not significantly affected by the large UV term. The spectrum becomes modified for larger $n$ such that $m_{n} / k$ approaches unity; in the absence of a UV brane curvature term, heavier modes have more significant overlap with the UV brane and are therefore more affected by this term, which tends to repel them from the UV brane.

Now we compute the normalization factors $N_{n}$. Taking $\kappa_{*}^{2}=1 /\left(2 M_{*}^{3}\right)$ we have

$$
\begin{aligned}
N_{n}^{2}=\int & d y e^{2 k y}\left\{J_{2}\left(\frac{m_{n}}{k} e^{k y}\right)+\beta_{n} Y_{2}\left(\frac{m_{n}}{k} e^{k y}\right)\right\}^{2} \\
& +\sum_{i} \frac{v_{i}}{2 k}\left\{J_{2}\left(\frac{m_{n}}{k} e^{k y_{i}}\right)+\beta_{n} Y_{2}\left(\frac{m_{n}}{k} e^{k y_{i}}\right)\right\}^{2} .
\end{aligned}
$$

In the LWS limit of $v_{0} \gg 1$, with $v_{L}=0$ again for simplicity, one has

$$
N_{n} \simeq \frac{e^{k L}}{\sqrt{2 k}}\left|J_{2}\left(z_{L}\right)\right|\left[1+\frac{e^{-2 k L}}{\left|J_{2}\left(z_{L}\right)\right|^{2}} \frac{1}{v_{0}}\right]^{1 / 2} \simeq \frac{e^{k L}}{\sqrt{2 k}}\left|J_{2}\left(z_{L}\right)\right|
$$

With this result one can consider the UV coupling of KK modes, the strength of which is sensitive to the UV value of the wavefunction: 6

$$
f_{n}(y=0) \simeq-\frac{\sqrt{2 k}}{e^{k L}} \frac{1}{\left|J_{2}\left(z_{L}\right)\right|} \frac{1}{v_{0}} \simeq-\frac{\sqrt{\pi}}{e^{k L / 2}} \frac{\sqrt{m_{n}}}{v_{0}},
$$

where we have made use of (18) for $m_{n} / k \ll 1$. Observe that the UV value is suppressed like $1 / v_{0} \ll 1$ and, in particular, one has $f_{n}(0)=0$ for $v_{0} \rightarrow \infty$. Thus, in the limit $M_{P l} \rightarrow \infty$, the KK gravitons are repelled from the UV brane. Despite this repulsion, the KK modes remain in the spectrum for $v_{0} \rightarrow \infty$.

\footnotetext{
${ }^{5}$ This is expected for $v_{L}=0$. Note that $v_{L} \neq 0$ will modify the IR BC 20], but not to the extent that the UV BC is modified by $v_{0} \gg 1$, due to the constraint $v_{L}<1$ that we obtain in Sec. 3 .

${ }^{6}$ The UV coupling of KK gravitons has previously been considered in the context of DGP gravity with a flat infinite extra dimension [23, 24].
} 
The spectrum of metric fluctuations also contains a massless spin-two mode and a massless scalar mode. We will discuss the scalar mode in detail in the next section. The profile for the spin-two zero mode is

$$
f_{0}(y)=e^{-2 k y} \sqrt{\frac{2 k}{1-e^{-2 k L}+\sum_{i} v_{i} e^{-2 k y_{i}}}},
$$

and, with this wavefunction, the zero mode is identified as the massless 4D graviton. This mode couples with strength $M_{P l}^{-1}$ and reproduces Einstein gravity. This coupling strength does not depend on the localization of the source field $T_{\mu \nu}$ and, to leading order, Einstein gravity is recovered for all fields in the warped space. Note that in the limit $v_{0} \rightarrow \infty$ one has $M_{P l} \rightarrow \infty$ and $f_{0}(y) \rightarrow 0$. Thus, in this limit the zero mode graviton is removed from the spectrum and $4 \mathrm{D}$ gravity is decoupled from the theory.

\section{The Little Radion}

As mentioned already, the spectrum of metric fluctuations also contains a massless scalar mode, namely the radion. In physical theories the radion must be massive if the length of the extra dimension is to be stabilized. We will detail an implementation of the GoldbergerWise mechanism for stabilizing the LWS in Sec. 5. However, it will first prove instructive to consider the massless radion. The radion only acquires its mass after the backreaction of the stabilizing dynamics is included [26], so the results obtained below for a massless radion will, in some instances, provide a good approximation for the calculable case of a weak backreaction.

We find it convenient to write the most general form of the metric, including background and scalar perturbations, as

$$
G_{M N}=\left(\begin{array}{cc}
a^{2}\left[\eta_{\mu \nu}+\nabla_{\mu} \nabla_{\nu} P_{3}+\eta_{\mu \nu}\left(2 P_{2}-a a^{\prime} P_{3}^{\prime}\right)\right] & 0 \\
0 & 1+2 P_{1}-\left(a^{2} P_{3}^{\prime}\right)^{\prime}
\end{array}\right)
$$

where $a(y)$ is the background warp factor, and $P_{1}, P_{2}$ and $P_{3}$ are spin-zero perturbations that depend on $x^{\mu}$ and $y$. This form of the metric is inspired by the gauge-invariant forms discussed in [27, 28]. With this parameterization, the Einstein equations have a particularly simple structure. Two of the bulk Einstein equations for the perturbations can be taken as

$$
\begin{array}{lll}
\partial_{\mu} \partial_{\nu}\left(P_{1}+2 P_{2}\right) & =0 & \mu \neq \nu, \\
\partial_{\mu}\left(\frac{a^{\prime}}{a} P_{1}-P_{2}^{\prime}\right) & =0 & \forall \mu .
\end{array}
$$

Taking the integration "constants" to be zero 7 the first equation relates $P_{2}$ to $P_{1}$, while the second determines the $y$-dependence of $P_{1}$. Using these results, the remaining bulk Einstein equation is simply $\square P_{1}=0$, which is satisfied for a massless $4 \mathrm{D}$ field. The perturbation $P_{3}$

\footnotetext{
${ }^{7}$ As required by the demand that all perturbations be localized in $x$.
} 
is completely free in the bulk and is a remnant gauge freedom 8 This is a generalization of the remnant gauge freedom in the massless sector described by the arbitrary function $F(y)$ in [18, 19], and physical quantities do not depend on $P_{3}$. The boundary conditions derive from the two additional boundary Einstein equations:

$$
P_{3}^{\prime}\left(y_{i}\right)=\frac{-v_{i}}{a\left(y_{i}\right)\left[\theta_{i} k a\left(y_{i}\right)+v_{i} a^{\prime}\left(y_{i}\right)\right]} P_{1}\left(y_{i}\right)
$$

Using the solutions to the above we can compute the effective 4D action for the physical scalar fluctuation. We perform separation of variables and solve for the profile of $P_{1}$, giving

$$
P_{1}=a^{-2}(y) \psi\left(x^{\mu}\right)
$$

This solution is consistent with the boundary conditions (26) for general $v_{i}$ so long as one chooses a $P_{3}$ with $P_{3}^{\prime} \neq 0$ at the boundaries. For the sources given in (2), the solution for the background metric is of the standard RS form, $a(y)=e^{-k y}$. Ignoring the $4 \mathrm{D}$ surface terms $\left(\psi\right.$ vanishes at $\left.x^{\mu} \rightarrow \infty\right)$, one can derive the action for the scalar perturbations up to quadratic order in $\psi$ as

$$
\mathcal{S}_{\mathcal{O}\left(\psi^{2}\right)}=\int d^{4} x\left[\frac{3 M_{*}^{3}}{k} e^{2 k L}\left(\frac{1}{1-v_{L}}-\frac{e^{-2 k L}}{1+v_{0}}\right)\right]\left(-\frac{1}{2} \eta^{\mu \nu} \partial_{\mu} \psi \partial_{\nu} \psi\right)
$$

It is worth noting that there are no terms linear in $\psi$, or additional quadratic terms, in the action (28). This provides an important check on the consistency of the calculation. In particular, the mass term 9 and higher-order derivative terms which appear at the quadratic level in individual terms in the action (2) cancel out in the full action. It is essential to include the Gibbons-Hawking term in the action to achieve this result.

The physical radion is defined as $r(x)=\psi(x) / N_{\psi}$, where $N_{\psi}$ is the following normalization constant:

$$
N_{\psi}^{2}=\frac{k}{3 M_{*}^{3}} e^{-2 k L} \frac{\left(1-v_{L}\right)\left(1+v_{0}\right)}{\left(1+v_{0}\right)-\left(1-v_{L}\right) e^{-2 k L}} .
$$

We see that the kinetic term is only well behaved for $v_{L}<1$, giving an upper bound on the size of the IR localized brane kinetic term. The UV term suffers no such constraint and one may safely take $v_{0} \gg 1$. In this limit one has

$$
N_{\psi}^{2} \simeq \frac{k}{3 M_{*}^{3}} e^{-2 k L}\left(1-v_{L}\right)\left[1+\frac{\left(1-v_{L}\right) e^{-2 k L}}{v_{0}}\right], \quad v_{0} \gg 1,
$$

and, to leading order, the dependence on $v_{0}$ drops out .

\footnotetext{
${ }^{8}$ See Appendix A for a discussion of the remaining gauge freedom in our chosen straight gauge.

${ }^{9}$ The radion will acquire mass once we specify a mechanism to stabilize the length of the extra dimension.
} 


\section{Little Radion Coupling to Matter}

In the previous section we detailed the physical gravi-scalar in the LWS (the "little radion" [4]). We would now like to determine the coupling of this mode to localized matter. The large UV curvature term modifies the wavefunction of the radion and is expected to alter its couplings. We will detail the dependence of the couplings on the UV term in this section. Our results will reduce to standard RS results in the limit $v_{i} \rightarrow 0$, allowing us to contrast the coupling of the little radion to the standard case with $v_{i}=0$.

The coupling of the radion to brane matter depends on the localization of the matter. To demonstrate our points we consider a scalar localized on the boundary at $y=y_{\phi}$ with action

$$
S_{\phi}=-\frac{1}{2} \int d^{5} x \sqrt{-g}\left\{g^{\mu \nu} \partial_{\mu} \phi \partial_{\nu} \phi+V(\phi)\right\} \delta\left(y-y_{\phi}\right)
$$

First consider the propagation of $\phi$ in the background metric. After integrating out the extra dimension the action is cast as

$$
S_{\phi}=-\frac{1}{2} \int d^{4} x\left\{\eta^{\mu \nu} \partial_{\mu} \phi \partial_{\nu} \phi+V(\phi)\right\}
$$

where we have rescaled $e^{-k y_{\phi}} \phi \rightarrow \phi$ to bring the kinetic term to a canonical form. Thus the dimensionful parameters in the potential $V(\phi)$ are "warped down" parameters, e.g. the mass in $V(\phi)$ is $m_{\phi}=e^{-k y_{\phi}} m_{0}$, where $m_{0}$ is the bare input mass parameter.

Now return to the original action (31) and expand the metric in terms of the scalar fluctuation as $g_{\mu \nu} \rightarrow g_{\mu \nu}+f_{\mu \nu}$, where $f_{\mu \nu}$ contains only the spin-zero parts of the perturbation. Integrating over the extra dimension, the action becomes

$$
\begin{aligned}
S_{\phi}=S_{0} & -\frac{1}{2} \int d^{4} x \sqrt{-g} f^{\mu \nu} \tilde{T}_{\mu \nu} \\
& -\frac{1}{8} \int d^{4} x \sqrt{-g}\left\{-f_{\nu}^{\mu} f_{\mu}^{\nu}+\frac{1}{2}\left(f_{\mu}^{\mu}\right)^{2}\right\}\left\{g^{\mu \nu} \partial_{\mu} \phi \partial_{\nu} \phi+V(\phi)\right\} \\
& +\int d^{4} x \sqrt{-g}\left(f_{\alpha}^{\mu} f^{\alpha \nu}-\frac{1}{2} f^{\mu \nu} f_{\alpha}^{\alpha}\right) \partial_{\mu} \phi \partial_{\nu} \phi+\ldots
\end{aligned}
$$

where

$$
\tilde{T}_{\mu \nu}=-\partial_{\mu} \phi \partial_{\nu} \phi+\frac{1}{2} g_{\mu \nu}\left\{g^{\alpha \beta} \partial_{\alpha} \phi \partial_{\beta} \phi+V(\phi)\right\}
$$

Rescaling $\phi$ to bring $S_{0}$ to a canonical form, we can write the term linear in the fluctuation as

$$
\left.S_{\phi}\right|_{\mathcal{O}(f)}=-\frac{1}{2} e^{2 k y_{\phi}} \int d^{4} x \eta^{\mu \alpha} \eta^{\nu \beta} f_{\mu \nu} T_{\alpha \beta}
$$

where $T_{\mu \nu}$ is now written in terms of canonical fields and the flat space metric; i.e. replace $g_{\mu \nu} \rightarrow \eta_{\mu \nu}$ in (34) to obtain $T_{\mu \nu}$. 
The gravi-scalar couples to $T_{\mu \nu}$ both with and without derivatives. Let us consider the non-derivative couplings:

$$
\left.S_{\phi}\right|_{\mathcal{O}(f)}=\frac{e^{2 k y_{\phi}}}{2}\left[1-\frac{v_{i} a^{\prime}}{a\left(\theta_{i} k a+v_{i} a^{\prime}\right)}\right] \int d^{4} x \psi T+\ldots
$$

where $T=\eta^{\mu \nu} T_{\mu \nu}$ and we have made use of Eqs. (24)-(27). We are interested primarily in the effects of the large UV term, so let us simply set $v_{L}=0$. Then the coupling of the physical radion is

$$
\left.S_{\phi}\right|_{\mathcal{O}(f)}=\frac{1}{2} \int d^{4} x \frac{r}{\Lambda_{r}} \times T+\ldots
$$

where the coupling is controlled by the dimensionful parameter

$$
\Lambda_{r}^{-1}=\sqrt{\frac{k}{3 M_{*}^{3}}} \sqrt{\frac{1+v_{0}}{1+v_{0}-e^{-2 k L}}} \times\left\{\begin{array}{ll}
e^{-k L} /\left(1+v_{0}\right) & y_{\phi}=0 \\
e^{k L} & y_{\phi}=L
\end{array} .\right.
$$

Let us consider two limits of the above expression. First, consider the RS limit of $v_{0} \rightarrow 0$ with $M_{*} \sim M_{P l}$ :

$$
\left.\Lambda_{r}^{-1}\right|_{\mathrm{RS}} \simeq \sqrt{\frac{k}{3 M_{*}^{3}}} \times \begin{cases}e^{-k L} & y_{\phi}=0 \\ e^{k L} & y_{\phi}=L\end{cases}
$$

Observe that the RS radion couples to UV localized fields with strength $\sim e^{-k L} / M_{*} \sim$ $e^{-k L} / M_{P l}$ and IR localized fields with strength $\sim 1 /\left(e^{-k L} M_{P l}\right) \sim e^{k L} / M_{*}$ (taking $k \sim M_{*}$ in both cases). The coupling to UV (IR) fields is thus suppressed (enhanced) relative to the bulk gravity scale $M_{*}$. Note that the IR coupling demonstrates the standard result that the radion couples to IR fields with a strength set by the inverse IR scale $\left(e^{-k L} M_{*}\right)^{-1}$ [29, 26, 30].

Next consider the LWS limit $M_{*} \ll M_{P l}$, which requires $v_{0} \gg 1$ to achieve a viable $4 \mathrm{D}$ Planck mass:

$$
\left.\Lambda_{r}^{-1}\right|_{\mathrm{LWS}} \simeq \sqrt{\frac{k}{3 M_{*}^{3}}} \times\left\{\begin{array}{ll}
e^{-k L} / v_{0} & y_{\phi}=0 \\
e^{k L} & y_{\phi}=L
\end{array} .\right.
$$

Observe that the coupling to IR fields is the same as that obtained in the RS limit, $\Lambda_{r} \sim$ $e^{-k L} M_{*}$. The large UV term does not modify the IR-brane coupling of the radion to leading order. For UV localized fields the radion coupling picks up a factor of $v_{0}^{-1} \ll 1$ and is significantly suppressed relative to the UV scale $M_{*}$ in the LWS limit. Note, however, that when expressed in terms of the 4D Planck mass, the coupling of the radion to UV matter remains as $\Lambda_{r} \sim e^{k L} M_{P l}$. Also observe that in the limit $v_{0} \rightarrow \infty$ the radion is repelled from the UV brane, while the IR coupling is unaffected. We will return to this feature in subsequent discussions.

Let us note that in the limit $v_{i} \rightarrow 0$ one can use the remaining gauge freedom to choose the form of the scalar fluctuations such that the derivative pieces in (23),$\nabla_{\mu} \nabla_{\nu} P_{3}$ and 
$P_{3}^{\prime}$, vanish. Thus, in the limit that the brane curvature terms are turned off, the standard parametrization of the scalar metric fluctuations in $\mathrm{RS}$ [29, 26, 30] is consistent with the boundary conditions. However, for $v_{i} \neq 0$ we find that one cannot remove the derivative pieces in (23) with a gauge choice and simultaneously obtain a solution that is consistent with the boundary conditions. We have checked this result using the metric parametrization of Refs. [18, 19] and arrive at the same conclusion.

\section{$5 \quad$ Stabilizing a Little Radius}

In the preceding sections we have detailed the spectrum of metric fluctuations in a LWS. In that analysis, however, no stabilizing dynamics were introduced so that the size of the extra dimension was not fixed and the radion remained massless. Realistic physical theories constructed on a LWS, like the Little RS model and the Mini-Seesaw, require the radion to develop a mass. Thus we must consider the effects of some stabilizing dynamics if we are to accurately report the spectrum of metric fluctuations in physically realistic cases.

We will follow the original idea of Goldberger and Wise [16] (also see [31]) and stabilize the little warped space using a bulk scalar $\Phi$. This produces a KK tower of physical scalars that contain an admixture of the KK modes of $\Phi$ and the gravi-scalar. As in the previous sections, we work with the interval approach [18, 19]. Essentially our goal in this section is to generalize the analysis of [18] to include a 5D scalar with bulk and brane potentials. The complete action is therefore

$$
\begin{aligned}
\mathcal{S}=\int_{\mathcal{M}} & d^{5} x \sqrt{-G}\left\{2 M_{*}^{3} \mathcal{R}-\frac{1}{2} G^{M N} \partial_{M} \Phi \partial_{N} \Phi-V(\Phi)\right\} \\
& +\sum_{i} \int d^{4} x \sqrt{-g}\left\{\frac{M_{*}^{3} v_{i}}{k} R-M_{*}^{3} k w_{i}-\frac{1}{4} t_{i} g^{\mu \nu} \partial_{\mu} \Phi \partial_{\nu} \Phi-\frac{1}{2} \lambda_{i}(\Phi)\right\} \\
& +4 M_{*}^{3} \oint_{\partial \mathcal{M}} \sqrt{-g} K .
\end{aligned}
$$

As before, $G_{M N}$ is the bulk metric and $g_{\mu \nu}$ the induced $4 \mathrm{D}$ boundary metric, with corresponding Ricci scalars $\mathcal{R}$ and $R$. We have allowed for brane kinetic terms in both the gravity $\left(v_{i}\right)$ and scalar $\left(t_{i}\right)$ sectors. $V$ is the bulk potential for the scalar $\Phi$, which now subsumes the bulk cosmological constant, and $\lambda_{i}$ are the brane localized potentials. The brane tensions $k w_{i}$ are explicitly separated from the brane potentials such that $\lambda_{i}(\Phi)=0$ for the background value of $\Phi$.

Let us emphasize that, in this section, we are not specifying the form of the background metric. The point is that the potentials $V(\Phi)$ and $\lambda_{i}(\Phi)$ will cause $\Phi$ to obtain a nonzero background value which, along with the bulk cosmological constant and the brane tensions, will source the metric. The analysis of this section will be performed for arbitrary potentials $V(\Phi)$ and $\lambda_{i}(\Phi)$, subject to the constraint that the metric preserves a 4D Lorentz symmetry. Ultimately we are interested in the case where the metric is a perturbed version of the standard RS form, in order to determine the mass of the little radion and to demonstrate 
stabilization in the LWS. We will specify a background solution appropriate for this case in the next subsection. However, our results in this section are completely general.

As usual, varying the above action with respect to the degrees of freedom gives the equations of motion. Varying with respect to the metric gives the bulk Einstein equations

$$
\left(\mathcal{R}_{M N}-\frac{1}{2} G_{M N} \mathcal{R}\right)-\frac{1}{4 M_{*}^{3}}\left(\partial_{M} \Phi \partial_{N} \Phi-\frac{1}{2} G_{M N} G^{P Q} \partial_{P} \Phi \partial_{Q} \Phi-G_{M N} V\right)=0
$$

and the boundary Einstein equations

$$
\begin{aligned}
& {\left[\frac{v_{i}}{k}\left(R_{\mu \nu}-\frac{1}{2} g_{\mu \nu} R\right)+\frac{1}{2} g_{\mu \nu} k w_{i}+\theta_{i} \sqrt{G^{55}}\left(g_{\mu \nu, 5}-g_{\mu \nu} g_{\alpha \beta, 5} g^{\alpha \beta}\right)\right.} \\
& \left.\quad-\frac{1}{4 M_{*}^{3}}\left(t_{i} \partial_{\mu} \Phi \partial_{\nu} \Phi-\frac{1}{2} t_{i} g_{\mu \nu} g^{\alpha \beta} \partial_{\alpha} \Phi \partial_{\beta} \Phi-g_{\mu \nu} \lambda_{i}\right)\right]_{y=y_{i}}=0 .
\end{aligned}
$$

Variation with respect to the field $\Phi$ gives the bulk Euler-Lagrange equation

$$
\partial_{M}\left(\sqrt{-G} G^{M N} \partial_{N} \Phi\right)-\sqrt{-G} V_{, \Phi}=0,
$$

where the subscript $\Phi$ on $V$ denotes a derivative. The corresponding boundary equations are

$$
\left[t_{i} \partial_{\mu}\left(\sqrt{-g} g^{\mu \nu} \partial_{\nu} \Phi\right)-\sqrt{-g} \lambda_{i, \Phi}-2 \theta_{i} \sqrt{-G} G^{5 N} \partial_{N} \Phi\right]_{y=y_{i}}=0 .
$$

Note that these boundary equations are expressed in straight gauge.

Our aim now is three-fold. We first solve for the background configuration of the above equations. This is straightforward; our solutions will be generic and expressed as a set of differential equations to be solved for a specific scalar potential. Following this we solve for first order spin-zero perturbations around the background, giving us the spectrum of scalar KK excitations. The answer will be in the form of a Schrödinger equation, whose potential depends on the background configuration from the first step. Third, we expand the original action to second order in the perturbations (expanded around the background) in order to compute the normalization condition for the KK modes.

Taking the usual warped metric ansatz:

$$
d s^{2}=a^{2}(y) \eta_{\mu \nu} d x^{\mu} d x^{\nu}+d y^{2},
$$

where the warp factor $a(y)$ is to be determined, and allowing the background value of $\Phi$ to depend only on $y$ [denoted as $\phi(y)]$, the background is solved by

$$
\begin{aligned}
0 & =\frac{a^{\prime \prime}}{a}-\frac{a^{\prime 2}}{a^{2}}+\frac{\phi^{\prime 2}}{12 M_{*}^{3}}, \\
0 & =24 M_{*}^{3} \frac{a^{\prime 2}}{a^{2}}-\frac{1}{2} \phi^{\prime 2}+V(\phi), \\
0 & =\left[w_{i} k-12 \theta_{i} \frac{a^{\prime}}{k a}\right]_{y=y_{i}}, \\
0 & =\left[\lambda_{i, \Phi}(\phi)+2 \theta_{i} \phi^{\prime}\right]_{y=y_{i}} .
\end{aligned}
$$


The first two equations relate bulk quantities, while the second two are required to satisfy the boundary equations. In this case, the fixed value of $w_{i}$ is the usual fine tuning for the RS brane tensions.

For the perturbations we use the same metric ansatz as in the non-stabilized case, Eq. (23), along with the scalar perturbation

$$
\Phi\left(x^{\mu}, y\right)=\phi(y)+P_{4}\left(x^{\mu}, y\right) .
$$

The $(\mu, \nu)$ and $(\mu, 5)$ bulk Einstein equations give

$$
\begin{aligned}
\partial_{\mu} \partial_{\nu}\left(P_{1}+2 P_{2}\right) & =0 & & \mu \neq \nu, \\
\partial_{\mu}\left(\frac{a^{\prime}}{a} P_{1}-P_{2}^{\prime}-\frac{a^{2} \phi^{\prime 2}}{24 M_{*}^{3}} P_{3}^{\prime}-\frac{\phi^{\prime}}{12 M_{*}^{3}} P_{4}\right) & =0 & & \forall \mu .
\end{aligned}
$$

The integration constants must be zero, similar to the non-stabilized case, allowing us to use the first equation to solve for $P_{2}$ and the second to solve for $P_{4}$. With these solutions, the remaining bulk equations reduce to a single equation for $P_{1}$, being

$$
-P_{1}^{\prime \prime}+\left(-2 \frac{a^{\prime}}{a}+2 \frac{\phi^{\prime \prime}}{\phi^{\prime}}\right) P_{1}^{\prime}+\left(4 \frac{a^{\prime}}{a} \frac{\phi^{\prime \prime}}{\phi^{\prime}}+\frac{\phi^{2}}{3 M_{*}^{3}}\right) P_{1}=\frac{1}{a^{2}} \square P_{1} .
$$

As in the non-stabilized case, the function $P_{3}$ is completely free in the bulk. There are four boundary equations for the perturbations. The first two are the same as in the non-stabilized case, Eq. (26) . The additional two result from the Euler-Lagrange boundary equations and are

$$
\left[\square P_{1}-\left(\frac{2 \theta_{i} a^{2} \phi^{\prime \prime}}{\phi^{\prime}}+a^{2} \lambda_{i, \Phi \Phi}(\phi)-\square t_{i}\right)\left(\frac{\theta_{i}\left(2 a^{\prime} P_{1}+a P_{1}^{\prime}\right)}{2 a}+\frac{v_{i} a \phi^{\prime 2} P_{1}}{24 M_{*}^{3}\left(k a+\theta_{i} v_{i} a^{\prime}\right)}\right)\right]_{y=y_{i}}=0 .
$$

In deriving this boundary condition we have used Eq. (54) evaluated at the boundaries (technically, at $y=\epsilon$ and $y=L-\epsilon$ for vanishing $\epsilon$ ).

To find solutions for $P_{1}$ we separate variables

$$
P_{1}\left(x^{\mu}, y\right)=p_{1}(y) \psi\left(x^{\mu}\right)
$$

with $\square \psi=m^{2} \psi$. Then, with an appropriate change of coordinates and rescaling of $p_{1}$, Eq. (54) becomes a Schrödinger equation. 10 This can be solved, along with the boundary conditions, to find the KK profiles $p_{1}(y)$ of the spin-zero perturbations, along with their corresponding mass eigenvalues $m^{2}$. In this way one obtains the physical spectrum of the theory.

We now turn to our third task, which is to compute the effective 4D action and find the normalization of each mode of the KK tower. Taking the original 5D action (41), we substitute in the metric ansatz (23), with separation of variables for $P_{1}$, and use the

\footnotetext{
${ }^{10}$ For the extension to multiple bulk scalars see Ref. [32].
} 
perturbation ansatz for $\Phi$ (51). We treat the action order by order in the perturbations, up to second order. Our answer takes the form

$$
\mathcal{S}=\int d^{4} x\left(\mathcal{L}^{(0)}+\mathcal{L}^{(1)}+\mathcal{L}^{(2)}\right)
$$

At each order the 4D Lagrangian density has a bulk piece, for which we shall attempt to do the $y$-integral, and the combined brane and boundary pieces.

For the zeroth order terms (that is, just the background) the Lagrangian is

$$
\mathcal{L}^{(0)}=\int_{0}^{L}\left(-16 M_{*}^{3} a^{2} a^{\prime 2}+\frac{1}{3} a^{4} \phi^{\prime 2}\right) d y+4 M_{*}^{3} \sum_{i} \theta_{i} a\left(y_{i}\right)^{3} a^{\prime}\left(y_{i}\right) .
$$

The first term comes from the bulk piece of (41) while the second is the combination of the brane and extrinsic curvature pieces. We have used the background equations to obtain this simplified form. One can show that the above integrand is equal to $-4 M_{*}^{3}\left(a^{3} a^{\prime}\right)^{\prime}$, and so the integral of these bulk terms exactly cancels the contributions from the brane and boundary pieces, making $\mathcal{L}^{(0)}$ vanish. This must be so for consistency; our background metric ansatz has Minkowski 4D slices and so there cannot be an effective 4D cosmological constant.

The first order terms reduce to

$$
\begin{aligned}
\mathcal{L}^{(1)}= & M_{*}^{3}\left[2 \int_{0}^{L} a^{2} p_{1} d y+\sum_{i} \frac{v_{i} a\left(y_{i}\right)^{3} p_{1}\left(y_{i}\right)}{k a\left(y_{i}\right)+\theta_{i} v_{i} a^{\prime}\left(y_{i}\right)}\right] \square \psi \\
& +\int_{0}^{L}\left[\left(-8 M_{*}^{3} a^{2} a^{\prime 2}+\frac{1}{6} a^{4} \phi^{\prime 2}\right) \square P_{3}\right] d y+2 M_{*}^{3} \sum_{i} \theta_{i} a\left(y_{i}\right)^{3} a^{\prime}\left(y_{i}\right) \square P_{3}\left(x^{\mu}, y_{i}\right) .
\end{aligned}
$$

To obtain this expression we have made use of the background equations as well as the first order equations for the $P_{i}$, and we have also done integration by parts (under the $y$-integral) on all terms that contain $y$-derivatives of $p_{1}$ and $P_{3}$. Since this Lagrangian appears under a $4 \mathrm{D}$ integral, and the perturbations are assumed to vanish at $4 \mathrm{D}$ infinity, the above expression does not contribute to the effective 4D action. This is again as expected.

Deriving the second order piece is a difficult task due to the large number of bulk, brane and boundary terms that must combine or cancel. The trick is to use the bulk background and perturbation equations, and perform integration by parts, to eliminate all derivatives of $a, \phi$ and $p_{1}$ that are second order or higher. This brings each term to a canonical form, allowing the surface terms coming from the $y$ integration by parts to combine or cancel with the brane and boundary terms. The factor $p_{1}^{\prime \prime}$ must be reduced in some cases by using integration by parts, and in other cases by using the differential Eq. (54). One also makes use of the boundary equation for $p_{1}$ to further simplify the resulting terms. By doing this, the second order terms can be brought into the expected form for a massive spin-zero field

$$
\mathcal{L}^{(2)}=\mathcal{N}\left(-\frac{1}{2} \eta^{\mu \nu} \partial_{\mu} \psi \partial_{\nu} \psi-\frac{1}{2} m^{2} \psi^{2}\right)
$$


where $m^{2}$ is the mass eigenvalue of the KK mode, and the normalization constant is

$$
\begin{aligned}
\mathcal{N}=6 & M_{*}^{3} \int_{0}^{L}\left(a^{2} p_{1}^{2}+24 M_{*}^{3} \frac{a^{\prime 2}}{\phi^{\prime 2}} p_{1}^{2}+24 M_{*}^{3} \frac{a a^{\prime}}{\phi^{\prime 2}} p_{1} p_{1}^{\prime}+6 M_{*}^{3} \frac{a^{2}}{\phi^{\prime 2}} p_{1}^{\prime 2}\right) d y \\
& +3 M_{*}^{3} \sum_{i} \frac{v_{i} a\left(y_{i}\right)^{3} p_{1}\left(y_{i}\right)^{2}}{k a\left(y_{i}\right)+\theta_{i} v_{i} a^{\prime}\left(y_{i}\right)} \\
& +\frac{1}{8} \sum_{i} t_{i}\left[12 M_{*}^{3} \theta_{i} \frac{2 a^{\prime}\left(y_{i}\right) p_{1}\left(y_{i}\right)+a\left(y_{i}\right) p_{1}^{\prime}\left(y_{i}\right)}{\phi^{\prime}\left(y_{i}\right)}+\frac{v_{i} a\left(y_{i}\right)^{2} \phi^{\prime}\left(y_{i}\right) p_{1}\left(y_{i}\right)}{k a\left(y_{i}\right)+\theta_{i} v_{i} a^{\prime}\left(y_{i}\right)}\right]^{2} .
\end{aligned}
$$

\subsection{The Radion Mass}

One can use the preceding results to compute the radion mass [i.e. the lightest KK mode of (54)] for a given background $\phi(y)$. We are interested in the case where the background geometry is of the standard RS form with a weak (calculable) perturbation due to the backreaction of the scalar field. We follow Ref. [26] and choose a perturbed background of the form

$$
\begin{aligned}
& a(y)=e^{-k y}\left(1-\frac{l^{2}}{6} e^{-2 u y}\right), \\
& \phi(y)=2 \sqrt{2} M_{*}^{3 / 2} l e^{-u y},
\end{aligned}
$$

which is valid in the $y \in[0, L]$ region. This corresponds to the potential $V(\Phi)=\left(W_{, \Phi}\right)^{2} / 2-$ $W^{2} / 6 M_{*}^{3}$ with $W(\Phi)=12 M_{*}^{3} k-u \Phi^{2} / 2$, along with the boundary potentials

$$
\lambda_{i}(\Phi)=-\theta_{i} W\left(\phi_{i}\right)-\theta_{i} W_{, \Phi}\left(\phi_{i}\right)\left(\Phi-\phi_{i}\right)+\gamma_{i}\left(\Phi-\phi_{i}\right)^{2},
$$

where $u, \phi_{i}$ and $\gamma_{i}$ are constants. In terms of the input parameters, the length of the extra dimension is now fixed at $L=u^{-1} \log \left(\phi_{0} / \phi_{L}\right)$, and the weak backreaction limit is defined by $\kappa_{*} \phi_{i} / \sqrt{2} \ll 1$. We work to order $l^{2}$ in the small parameter $l=\kappa_{*} \phi_{0} / \sqrt{2}$ in this section 11 The solution for $p_{1}(y)$ will be a perturbed form of the massless solution

$$
p_{1}(y)=e^{2 k y}\left\{1+l^{2} f(y)\right\} .
$$

The bulk equation for $f$ turns out to be the same as the case without brane curvature terms, namely [26]

$$
f^{\prime \prime}+2(k+u) f^{\prime}=\frac{4}{3} u(u-k) e^{-2 u y}-\tilde{m}^{2} e^{2 k y},
$$

where $m^{2}=l^{2} \tilde{m}^{2}$ (the mass of the radion is on the order of the correction to the background). Note that the backreaction must be included to obtain a non-zero radion mass. The solution in the bulk is 26 ]

$$
f^{\prime}(y)=-\frac{2}{3} u\left(1-\frac{u}{k}\right) e^{-2 u y}-\tilde{m}^{2} \frac{1}{4 k+2 u} e^{2 k y}+A e^{-2(k+u) y},
$$

\footnotetext{
${ }^{11}$ The expression for $\phi$ is actually correct to all orders in $l$.
} 
where $A$ is an integration constant.

For the boundary condition (55) we shall work in the limit of stiff brane potentials, $\lambda_{i, \Phi \Phi} \rightarrow \infty$. Then for our perturbed background we have

$$
\left[f^{\prime}+\frac{2}{3} u e^{-2 u y}+\frac{2}{3} \frac{u^{2}}{k} e^{-2 u y} \frac{\theta_{i} v_{i}}{1-\theta_{i} v_{i}}\right]_{y=y_{i}}=0
$$

Using the solution for the radion in the bulk, Eq. (67), we can obtain the mass of the lightest KK spin-zero state

$$
m^{2}=\frac{4 l^{2}}{3} \frac{(2 k+u) u^{2}}{k}\left(\frac{1}{1-v_{L}}-\frac{e^{-2 k L}}{1+v_{0}}\right)\left(e^{2(k+u) L}-e^{-2 k L}\right)^{-1} .
$$

This expression for the radion mass generalizes the result of Ref. [26] for the case of non-zero brane curvature terms, $v_{i} \neq 0$. Furthermore, in the limit $v_{i} \rightarrow 0$ it reduces to the result in [26], as expected. For $v_{L}=0$, we observe that the difference between $v_{0}=0$ and $v_{0} \rightarrow \infty$ is negligible. Therefore the large UV brane term required to include viable 4D gravity on a LWS does not significantly affect the mass of the radion and the LWS is suitably stabilized via the Goldberger-Wise mechanism. Equation (69) also demonstrates the sensitivity of the radion mass to an IR localized brane curvature term. Observe that values of the coefficient $v_{L}$ in the range $120<v_{L}<1$ tend to increase the mass of the radion relative to the standard RS result.

To determine the coupling of the radion to matter in the stabilized LWS we need to evaluate the normalization constant $\mathcal{N}$ in Eq. (61). We find

$$
\mathcal{N}=\frac{3 M_{*}^{3}}{k} e^{2 k L}\left(\frac{1}{1-v_{L}}-\frac{e^{-2 k L}}{1+v_{0}}\right)+\mathcal{O}\left(l^{2}\right),
$$

which, to leading order, is the same as that calculated in the non-stabilized case, Eq. (28). With this result one can redo the calculations of Sec. 4 to determine the coupling of the radion to matter. To leading order the couplings agree with those obtained in Sec. 4 ,

\section{Applications}

In this section we briefly discuss some applications of our results.

\subsection{Little Randall-Sundrum Model}

The little RS model attempts to address the SM gauge-hierarchy problem by employing a LWS with IR (UV) scale of order $\mathrm{TeV}\left(M_{*} \sim 10^{3} \mathrm{TeV}\right)$ [2]. It provides a candidate UV completion for the SM up to energies of order $10^{3} \mathrm{TeV}$. In order to be a completely realistic

\footnotetext{
${ }^{12}$ Recall that consistency demands $v_{L}<1$.
} 
effective theory for energies $E<M_{*}$, the LRS model should include 4D Einstein gravity. Though this was not considered in previous constructs, our results show that by introducing a large UV brane curvature term, 4D gravity can be consistently incorporated into this effective theory framework. In addition to incorporating 4D Einstein gravity, this approach modifies the wavefunctions of the metric fluctuations. However, from a phenomenological perspective, this modification may be unimportant for the KK gravitons; the modification occurs in the vicinity of the UV brane, while the KK gravitons are strongly localized towards the IR. One therefore expects the coupling of KK gravitons to SM fields to be essentially the same as that in [2], where a simple Dirichlet UV BC was employed for the KK gravitons.

On the other hand, we have seen that the wavefunction of the radion is also modified by the large UV-brane curvature term. Previous works have employed the standard RS parametrization for the radion fluctuation to study the phenomenology of this mode in the LRS framework [4]. As we have seen, the modification that leads to a Dirichlet BC for the KK gravitons also modifies the radion wave function so that, strictly speaking, consistency demands that the radion's wave function should also be modified in LRS studies employing a Dirichlet UV BC for the KK gravitons. The question then arises, is this modification important in terms of the coupling and the phenomenology? The radion couples like a dilaton and thus couples to the trace of the stress-energy tensor. For on-shell fields, this trace is (classically) determined by the dimensionful parameters in the Lagrangian. Thus the coupling of the radion to on-shell SM fermions and massive gauge bosons occurs locally on the TeV brane, where the Higgs, which breaks both electroweak symmetry and the classical scale invariance of the SM, is localized. In Sec. 4 we saw that the coupling of the radion to IR localized sources was not modified by the large UV curvature term, relative to the usual RS result. Thus we expect the radion coupling to on-shell SM fields to be well approximated by the standard parametrization, even when gravity is included 13 Using our results, one can show that the form of the radion coupling to the zero mode of a massless bulk vector (for example, the photon or gluon) matches the form used in previous analysis [4], up to corrections suppressed by $v_{0}$.

\subsection{A Mini Seesaw in a Little Warped Space}

A different application of a LWS was presented in [5, 6]. In these works, the SM was localized on the UV brane of a LWS whose UV (IR) scale was $M_{*} \sim \mathrm{TeV}\left(e^{-k L} M_{*} \sim \mathrm{GeV}\right)$, with sterile neutrinos propagating in the bulk. It was shown that realistic theories of neutrino mass can be constructed in this framework, without recourse to any high energy (supra-TeV) scale [5]. Our present results show that, by introducing a UV localized Einstein-Hilbert term with large coefficient, 4D gravity can be consistently included in this low-energy framework. Furthermore, the current results clear up some potential concerns regarding the strength with which the KK gravitons couple to the UV localized SM. We have seen in Sec. 2 and Sec. 4 that, respectively, the coupling of the KK gravitons and the radion to UV brane fields is suppressed by the large UV brane term. In both cases the coupling to the UV brane goes like $1 / v_{0} \ll 1$. Thus we conclude that once low-energy $4 \mathrm{D}$ gravity is included in the model

\footnotetext{
${ }^{13} \mathrm{~A}$ detailed discussion of the radion coupling to bulk on-shell fields can be found in [33].
} 
of [5, 6], the couplings of the SM to the KK gravitons and the radion are negligible small and, in particular, the model does indeed provide a consistent, viable, low-energy theory. Furthermore, we note that the suppressed coupling of the radion to the UV localized SM has a potentially interesting consequence. If the radion is lighter than the lightest sterile neutrino, the only available decay channels would be to lighter SM fields. Given the weakness of the direct coupling to the SM, the radion may be sufficiently long lived to provide a dark matter candidate. It would be interesting to consider this possibility in detail.

\section{Comments on AdS/CFT}

Via the AdS/CFT correspondence [34], RS models are thought to be dual to strongly coupled $4 \mathrm{D}$ theories that are (approximately) conformal for energies $M_{*}>E>e^{-k L} M_{*}$ [35]. The conformal symmetry is broken explicitly in the UV by a cutoff (dual to the UV brane) and spontaneously in the IR (dual to the IR brane). UV (IR) localized fields in the 5D picture are dual to fundamental (composite) fields in the 4D theory (roughly; see e.g. [36]). Thus the zero-mode graviton, which is localized towards the UV brane, is dual to a fundamental $4 \mathrm{D}$ graviton that is external to the CFT. The massive KK-gravitons and the radion are, respectively, dual to spin-two composites and a spin-zero composite dilaton. From the perspective of the dual 4D theory, a number of features detailed above are readily understood.

In standard RS models the warping generates the weak/Planck hierarchy and the UV scale is of order the Planck scale; the UV scale is thus associated with the need to cutoff the CFT to include 4D Einstein gravity. In the absence of a UV localized Einstein-Hilbert term, the 4D Planck mass is generated entirely by the dynamics of the (cut-off) CFT. That is, the usual expression for the Planck mass in RS:

$$
M_{P l}^{2} \sim \frac{M_{*}^{3}}{k}
$$

is dual to an induced Planck mass that results from loop 14 containing CFT modes [37]. From the perspective of the dual 4D theory there is no particular reason why the 4D Planck mass should be entirely induced by the CFT and, more generally, the 4D Planck mass could contain a "bare" contribution that arises either from integrating out heavy fields present in the UV completion or as a fundamental input in the theory. Irrespective of the details of its origin, a bare contribution to the Planck mass in the $4 \mathrm{D}$ theory is consistent with the symmetries of the theory and, in the standard effective-theory approach, is expected to be present. The inclusion of a bare contribution to the Planck mass in the dual 4D theory is dual to including a UV-localized Einstein-Hilbert term in the 5D theory. In this case the 4D Planck mass is:

$$
M_{P l}^{2} \sim \frac{M_{*}^{3}}{k}+M_{U V}^{2}=\frac{M_{*}^{3}}{k}\left(1+v_{0}\right) .
$$

In the LWS limit one has $M_{*} \ll M_{P l}$ and $v_{0} \gg 1$ is necessary to include 4D Einstein gravity, giving $M_{P l}^{2} \sim\left(M_{*}^{3} / k\right) v_{0}$. In the dual formulation it is clear why it is consistent to include a

\footnotetext{
${ }^{14}$ Strictly, these may be tree-level diagrams with external graviton legs, rather than loops.
} 
large UV Einstein-Hilbert term, $M_{U V} \sim M_{P l}$, despite the fact that the UV cutoff is much less than the Planck scale - this is simply the standard low-energy effective theory approach for including gravity. The theory breaks down at energies $M_{*} \ll M_{P l}$ and requires UV completion, but also contains a consistent description of gravity for distances $>1 / M_{*}$.

It is also clear why the KK graviton spectrum is relatively insensitive to the UV localized term. Including the UV term is equivalent to modifying the fundamental sector of the dual theory. The spectrum of spin-two composites of the CFT should not be significantly perturbed when the properties of fields that are external to the CFT (fundamentals) are altered. Similarly, the strength with which the composite dilaton interacts with other CFT modes should not depend on the details of the fundamental sector, which is consistent with the fact that the radion-IR-brane coupling is insensitive to the large UV term.

Sending the UV term to infinity, $v_{0} \rightarrow \infty$, makes the effective 4D Planck mass infinite and decouples the massless graviton from the particle spectrum. This modification of the fundamental sector should not remove composite modes from the spectrum, consistent with the finding that the radion and the KK gravitons remain in the spectrum in the limit $v_{0} \rightarrow \infty$. In the dual theory, interactions between fundamental fields and the CFT are mediated by gravity. Thus, in the limit that the massless graviton is removed, the two sectors decouple, in agreement with our observation that the coupling of KK gravitons and the radion to UV fields goes to zero for $v_{0} \rightarrow \infty$.

\section{Conclusion}

We have studied the consistent inclusion of low-energy Einstein gravity in theories constructed on a Little Warped Space; i.e. a truncated slice of $A d S_{5}$ with a bulk gravity scale that is much less than the $4 \mathrm{D}$ Planck mass, $M_{*} \ll M_{P l}$. To provide completely realistic lowenergy theories, models constructed on such spaces should reproduce 4D Einstein gravity for energies $E<M_{*}$. The approach we have detailed provides a consistent description of low-energy gravity by including a large UV-localized Einstein-Hilbert term (equivalently, a DGP term). The presence of such a term can be motivated by string-theoretic realizations of the RS model, in which a parametrically large UV curvature term can arise [15]. In addition to realizing 4D Einstein gravity, we have detailed the gravitational sector of the Little Warped Space, including both the KK graviton spectrum and the properties of the radion. Furthermore, we have shown that Goldberger-Wise stabilization can be successfully implemented in these spaces, and obtained the dependence of the radion mass and couplings on the brane localized curvature. These ingredients will play a role in any theory constructed on a LWS 15

Our results demonstrate that realistic low energy theories can be constructed on Little Warped Spaces and, in particular, have relevance for existing models in the literature [2,

\footnotetext{
${ }^{15}$ Models constructed on a LWS will require stabilization, though other possibilities exist beyond the Goldberger-Wise approach. However, the stabilization of the extra dimension in string realizations of RS models is well modeled by the Goldberger-Wise method [15, providing further motivation for this approach.
} 
5, 6. Given that large UV-localized curvature terms arise in string realizations of the RS model [15], we believe that the study of phenomenological models on Little Warped Spaces is well motivated - both within the context of solutions to the SM gauge-hierarchy problem, as in the Little RS model [2], and the study of warped hidden sectors, as in the MiniSeesaw [5, 6].

\section{Acknowledgements}

DG was supported by the Netherlands Foundation for Fundamental Research of Matter (FOM), and the Netherlands Organisation for Scientific Research (NWO), and thanks the MPIK (Heidelberg) for hospitality. KM thanks H. Davoudiasl and is grateful to Nikhef for hospitality.

\section{Appendix}

\section{A Gauge Freedom with the Variables $P_{i}$}

The action of a general coordinate transformation $x^{M} \rightarrow x^{M}+\xi^{M}(x, y)$ on an arbitrary metric perturbation $h_{M N}$ is

$$
\begin{aligned}
h_{\mu \nu} & \rightarrow h_{\mu \nu}-\nabla_{\mu} \xi_{\nu}-\nabla_{\nu} \xi_{\mu}-g_{\mu \nu} \frac{2 a^{\prime}}{a} \xi^{5} \\
h_{\mu 5} & \rightarrow h_{\mu 5}-g_{\mu \nu} \xi^{\nu \prime}-\partial_{\mu} \xi^{5} \\
h_{55} & \rightarrow h_{55}-2 \xi^{5^{\prime}} .
\end{aligned}
$$

One can always perform a gauge transformation to bring the perturbation to a straight gauge with the form of Eq. (23). Note that a straight gauge is defined by the demand that (i) the $(M, N)=(\mu, 5)$ components of the metric vanish: $G_{\mu 5}=G_{\mu 5}^{0}+h_{\mu 5}=0$, where $G_{M N}^{0}$ is the background metric; and that $(i i)$ the gauge parameters $\xi^{5}(x, y)$ vanish at the boundaries, $\xi^{5}\left(x, y_{i}\right)=0$ for $y_{i}=0, L[18$. The latter restriction ensures that the boundaries are located at the slices $y_{i}=0, L$, and removes the pure gauge brane-bending mode from the spectrum [18].

Starting with an arbitrary $h_{55}$, one obtains the form used in (23) by performing a gauge transformation with parameters $\xi^{\mu}(x, y)=0$ and:

$$
\xi^{5}(x, y)=\frac{1}{2} \int_{0}^{y} d \tilde{y}\left(h_{55}-2 P_{1}\right)+\frac{1}{2}\left[a^{2} P_{3}^{\prime}\right]_{0}^{y},
$$

subject to the constraint:

$$
\int_{0}^{L} d \tilde{y} P_{1}-\frac{1}{2}\left[a^{2} P_{3}^{\prime}\right]_{0}^{L}=\frac{1}{2} \int_{0}^{L} d \tilde{y} h_{55},
$$


in accordance with the definition of a straight gauge. Note that, if the boundary conditions require $P_{3}^{\prime} \propto P_{1}$, as found in the text [see Eq. (26) ], we can write $P_{1}=p_{1}(y) \psi(x)$ and obtain

$$
\psi(x)=\frac{\frac{1}{2} \int_{0}^{L} d \tilde{y} h_{55}}{\int_{0}^{L} d \tilde{y} p_{1}-\frac{1}{2}\left[a^{2} b_{i} v_{i} p_{1}\right]_{0}^{L}},
$$

as the definition for $\psi$, where $b_{i}$ are constants. Thus, for arbitrary $h_{55}$, the denominator should be nonzero. One completes the transition to a straight gauge by performing a second gauge transformation with $\xi^{5}(x, y)=0$ and $\xi^{\mu}(x, y)$ such that:

$$
h_{\mu 5}-2 g_{\mu \nu} \xi^{\nu \prime}=0
$$

The form of the $(M, N)=(\mu, \nu)$ components of the perturbation can then always be cast as in Eq. (23).

Further gauge transformations will preserve the straight-gauge form provided the gauge parameters $\xi^{M}$ satisfy

$$
\partial_{\mu} \xi^{5}(x, y)=-g_{\mu \nu} \partial_{5} \xi^{\nu}(x, y)
$$

which gives

$$
\xi^{\mu}(x, y)=-\int^{y} d \tilde{y} g^{\mu \nu} \partial_{\nu} \xi^{5}(x, \tilde{y})+\chi^{\mu}(x) .
$$

Thus, in a general straight gauge one has a remaining gauge choice described by the arbitrary functions $\xi^{5}(x, y)$ and $\chi^{\mu}(x)$, with $\xi^{\mu}(x, y)$ fixed as above and $\xi^{5} \mid=0$. Note that, because we have not chosen $h_{55}$ to be of separable form, we presently have more remnant gauge freedom than [18] (see Eq. (226) in the published version). Specifically, once $h_{55}$ is chosen separable, the $x$-dependence of $\xi^{5}(x, y)$ is fixed.

In a straight gauge, the transformation (73) becomes

$$
\begin{aligned}
h_{\mu \nu} & \rightarrow h_{\mu \nu}-\nabla_{\mu} \chi_{\nu}(x)-\nabla_{\nu} \chi_{\mu}(x)-g_{\mu \nu} \frac{2 a^{\prime}}{a} \xi^{5}(x, y)+2 a^{2} \nabla_{\mu} \nabla_{\nu} \int^{y} d \tilde{y} \frac{\xi^{5}(x, \tilde{y})}{a^{2}}, \\
h_{\mu 5} & \rightarrow h_{\mu 5}, \\
h_{55} & \rightarrow h_{55}-2 \partial_{5} \xi^{5}(x, y) .
\end{aligned}
$$

Consider the action of a gauge transformation on the variables $P_{i}$ employed in the text. Let us ignore the $4 \mathrm{D} \chi^{\mu}(x)$ general coordinate transformation and concentrate on the $\xi^{5}(x, y)$ gauge freedom. If we choose

$$
\begin{aligned}
& P_{1} \rightarrow P_{1} \\
& P_{2} \rightarrow P_{2} \\
& P_{3} \rightarrow P_{3}+2 \int^{y} d \tilde{y} \frac{\xi^{5}(x, \tilde{y})}{a^{2}}
\end{aligned}
$$

then from (23) we have

$$
\begin{aligned}
h_{\mu \nu} & =a^{2} \nabla_{\mu} \nabla_{\nu} P_{3}+a^{2} \eta_{\mu \nu}\left(2 P_{2}-a a^{\prime} P_{3}^{\prime}\right) \\
& \rightarrow h_{\mu \nu}-g_{\mu \nu} \frac{2 a^{\prime}}{a} \xi^{5}(x, y)+2 a^{2} \nabla_{\mu} \nabla_{\nu} \int^{y} d \tilde{y} \frac{\xi^{5}(x, \tilde{y})}{a^{2}}
\end{aligned}
$$


and

$$
\begin{aligned}
h_{55} & =2 P_{1}-\left(a^{2} P_{3}^{\prime}\right)^{\prime} \\
& \rightarrow 2 P_{1}-\partial_{5}\left[a^{2}\left(P_{3}^{\prime}+\frac{2 \xi^{5}(x, y)}{a^{2}}\right)\right] \\
& =h_{55}-2 \partial_{5} \xi^{5}(x, y) .
\end{aligned}
$$

This is the correct transformation of the metric perturbations, which tells us that the transformation (81) correctly encapsulates the remaining gauge freedom. Thus, $P_{1}$ and $P_{2}$ remain unchanged under a gauge transformation with non-zero $\xi^{5}(x, y)$, while $P_{3}$ changes as above.

It would seem that one can choose $\xi^{5}(x, y)$ such that $P_{3}$ is gauged from the spectrum. However, this is not necessarily the case. Noting that under a gauge transformation one has:

$$
P_{3}^{\prime} \rightarrow P_{3}^{\prime}+\frac{2 \xi^{5}(x, y)}{a^{2}}
$$

and that straight gauges demand $\xi^{5} \mid=0$, we see that $P_{3}^{\prime}$ can only be gauged away when $P_{3}^{\prime} \mid=0$. For $b_{i} \neq 0$, as found in the text, this is only true for $v_{i}=0$.

One can use the remaining gauge freedom to relate $P_{3}^{\prime}$ to $P_{1}$ by specifying the $x$ dependence of $\xi^{5}$ such that

$$
P_{3}^{\prime}(x, y) \rightarrow F_{3}(y) P_{1}(x, y),
$$

with $F_{3}(y)$ satisfying $F_{3} \mid=b_{i} v_{i}$ but otherwise arbitrary. The remaining gauge freedom, beyond the $4 \mathrm{D}$ general coordinate transformations, is then fixed by specifying the $y$-dependence of $\xi^{5}$ to determine $F_{3}$.

\section{References}

[1] L. Randall and R. Sundrum, Phys. Rev. Lett. 83, 3370 (1999) arXiv:hep-ph/9905221.

[2] H. Davoudiasl, G. Perez and A. Soni, Phys. Lett. B 665, 67 (2008) arXiv:0802.0203 [hep-ph]].

[3] M. Bauer, S. Casagrande, L. Grunder, U. Haisch, M. Neubert, Phys. Rev. D79, 076001 (2009) [arXiv:0811.3678 [hep-ph]]; H. Davoudiasl, S. Gopalakrishna, A. Soni, Phys. Lett. B686, 239-243 (2010) arXiv:0908.1131 [hep-ph]].

[4] H. Davoudiasl, T. McElmurry, A. Soni, Phys. Rev. D82, 115028 (2010) arXiv:1009.0764 [hep-ph]].

[5] K. L. McDonald, Phys. Lett. B696, 266-272 (2011) arXiv:1010.2659 [hep-ph]].

[6] M. Duerr, D. P. George, K. L. McDonald, arXiv:1105.0593 [hep-ph]. 
[7] K. L. McDonald, Phys. Rev. D77, 124046 (2008) arXiv:0804.0654 [hep-th]].

[8] G. R. Dvali, G. Gabadadze, M. Porrati, Phys. Lett. B485, 208-214 (2000) hep-th/0005016].

[9] A. Nicolis, R. Rattazzi, JHEP 0406, 059 (2004) hep-th/0404159.

[10] D. Gorbunov, K. Koyama, S. Sibiryakov, Phys. Rev. D73, 044016 (2006) hep-th/0512097.

[11] A. Adams, N. Arkani-Hamed, S. Dubovsky, A. Nicolis, R. Rattazzi, JHEP 0610, 014 (2006) hep-th/0602178.

[12] C. Charmousis, R. Gregory, N. Kaloper, A. Padilla, JHEP 0610, 066 (2006) hep-th/0604086]; R. Gregory, N. Kaloper, R. C. Myers, A. Padilla, JHEP 0710, 069 (2007) [arXiv:0707.2666 [hep-th]].

[13] M. A. Luty, M. Porrati, R. Rattazzi, JHEP 0309, 029 (2003) hep-th/0303116.

[14] H. L. Verlinde, Nucl. Phys. B580, 264-274 (2000) hep-th/9906182]; I. R. Klebanov, A. A. Tseytlin, Nucl. Phys. B578, 123-138 (2000) [hep-th/0002159]; I. R. Klebanov, M. J. Strassler, JHEP 0008, 052 (2000) arXiv:hep-th/0007191 [hep-th]]; S. B. Giddings, S. Kachru, J. Polchinski, Phys. Rev. D66, 106006 (2002) hep-th/0105097; S. Kachru, R. Kallosh, A. D. Linde, S. P. Trivedi, Phys. Rev. D68, 046005 (2003) hep-th/0301240]; A. Hebecker, J. March-Russell, Nucl. Phys. B781, 99-111 (2007) hep-th/0607120]; B. Hassanain, J. March-Russell, M. Schvellinger, JHEP 0710, 089 (2007) [arXiv:0708.2060 [hep-th]].

[15] F. Brummer, A. Hebecker, E. Trincherini, Nucl. Phys. B738, 283-305 (2006) hep-th/0510113.

[16] W. D. Goldberger and M. B. Wise, Phys. Rev. Lett. 83, 4922 (1999) [hep-ph/9907447].

[17] Z. Lalak, R. Matyszkiewicz, JHEP 0111, 027 (2001) hep-th/0110141.

[18] M. S. Carena, J. D. Lykken, M. Park, Phys. Rev. D72, 084017 (2005) hep-ph/0506305.

[19] R. Bao, M. S. Carena, J. Lykken, M. Park, J. Santiago, Phys. Rev. D73, 064026 (2006) hep-th/0511266.

[20] H. Davoudiasl, J. L. Hewett, T. G. Rizzo, JHEP 0308, 034 (2003) hep-ph/0305086.

[21] H. Davoudiasl, B. Lillie, T. G. Rizzo, JHEP 0608, 042 (2006) hep-ph/0508279.

[22] G. W. Gibbons, S. W. Hawking, Phys. Rev. D15, 2752-2756 (1977).

[23] G. R. Dvali, G. Gabadadze, M. Kolanovic, F. Nitti, Phys. Rev. D64, 084004 (2001) hep-ph/0102216.

[24] G. R. Dvali, G. Gabadadze, M. Kolanovic, F. Nitti, Phys. Rev. D65, 024031 (2002) hep-th/0106058. 
[25] H. Davoudiasl, J. L. Hewett, T. G. Rizzo, Phys. Rev. Lett. 84, 2080 (2000) hep-ph/9909255].

[26] C. Csaki, M. L. Graesser, G. D. Kribs, Phys. Rev. D63, 065002 (2001) hep-th/0008151.

[27] H. A. Bridgman, K. A. Malik, D. Wands, Phys. Rev. D65, 043502 (2002) astro-ph/0107245.

[28] C. Deffayet, Phys. Rev. D66, 103504 (2002) hep-th/0205084.

[29] W. D. Goldberger, M. B. Wise, Phys. Lett. B475, 275-279 (2000) arXiv:hep-ph/9911457 [hep-ph]].

[30] K. -m. Cheung, Phys. Rev. D63, 056007 (2001) hep-ph/0009232]; C. Charmousis, R. Gregory, V. A. Rubakov, Phys. Rev. D62, 067505 (2000) [hep-th/9912160]; T. Tanaka, X. Montes, Nucl. Phys. B582, 259-276 (2000) |hep-th/0001092]; J. Garriga, O. Pujolas, T. Tanaka, Nucl. Phys. B605, 192-214 (2001) [hep-th/0004109]; L. Kofman, J. Martin, M. Peloso, Phys. Rev. D70, 085015 (2004) hep-ph/0401189]; C. Csaki, J. Hubisz, S. J. Lee, Phys. Rev. D76, 125015 (2007) arXiv:0705.3844 [hep-ph]]; Y. Eshel, S. J. Lee, G. Perez, Y. Soreq, arXiv:1106.6218 [hep-ph].

[31] J. Garriga and A. Pomarol, Phys. Lett. B 560, 91 (2003) arXiv:hep-th/0212227 O. DeWolfe, D. Z. Freedman, S. S. Gubser, A. Karch, Phys. Rev. D62, 046008 (2000) hep-th/9909134.

[32] S. M. Aybat, D. P. George, JHEP 1009, 010 (2010) arXiv:1006.2827 [hep-th]].

[33] T. G. Rizzo, JHEP 0206, 056 (2002) hep-ph/0205242].

[34] J. M. Maldacena, Adv. Theor. Math. Phys. 2, 231 (1998) [Int. J. Theor. Phys. 38, 1113 (1999)] arXiv:hep-th/9711200]; S. S. Gubser, I. R. Klebanov and A. M. Polyakov, Phys. Lett. B 428, 105 (1998) [arXiv:hep-th/9802109]; E. Witten, Adv. Theor. Math. Phys. 2, 253 (1998) |arXiv:hep-th/9802150|.

[35] N. Arkani-Hamed, M. Porrati and L. Randall, JHEP 0108 (2001) 017 arXiv:hep-th/0012148]; R. Rattazzi and A. Zaffaroni, JHEP 0104, 021 (2001) arXiv:hep-th/0012248].

[36] B. Batell, T. Gherghetta, Phys. Rev. D76, 045017 (2007) [arXiv:0706.0890 [hep-th]].

[37] K. Agashe, A. Delgado, Phys. Rev. D67, 046003 (2003) hep-th/0209212]. 